Journal of Transport and Land Use 1:1 (Summer 2008) pp. 21-39

Available at http://jtlu.org

JTLU

\title{
Cities as organisms: Allometric scaling of urban road networks
}

\author{
Horacio Samaniego \\ Instituto de Silvicultura, Universidad Austral de Chile ${ }^{\text {a }}$ \\ Melanie E. Moses \\ University of New Mexico ${ }^{b}$
}

\begin{abstract}
Just as the cardiovascular network distributes energy and materials to cells in an organism, urban road networks distribute energy, materials and people to locations in cities. Understanding the topology of urban networks that connect people and places leads to insights into how cities are organized. This paper proposes a statistical approach to determine features of urban road networks that affect accessibility. Statistics of road networks and traffic patterns across 425 U.S. cities show that urban road networks are much less centralized than biological vascular networks. As a result, per capita road capacity is independent of the spatial extent of cities. In contrast, driving distances depend on city area, although not as much as is predicted by a completely centralized model. This intermediate pattern between centralized and decentralized extremes may reflect a mixture of different travel behaviors. The approach presented here offers a novel macroscopic perspective on the differences between small and large cities and on how road infrastructure and traffic might change as cities grow.
\end{abstract}

Keywords: Allometric scaling, urban form, Metabolic Scaling Theory, network growth

\section{Introduction}

Access to destinations (i.e. opportunities such as jobs, stores, recreational locations, etc.) is largely determined by how efficiently the underlying road network facilitates the flow of people to their destinations. Access is highly constrained by the topology of the network and to some extent by the form of transportation employed to traverse it. A variety of definitions of accessibility have been proposed (e.g. Hansen 1959; Horner 2004; Krizek 2005; Kwan et al. 2003), but all of these estimates of accessibility consider how destinations and residents are distributed within the city. Therefore, it

\footnotetext{
ahoracio.samaniego@gmail.com

${ }^{b}$ melaniem@cs.unm.edu
}

Copyright 2008 Horacio Samaniego and Melanie E. Moses.

Licensed under the Creative Commons Attribution - NonCommercial License 3.0. 
is important to assess how the network topology connects residents to destinations in order to improve the efficiency of road networks.

Accessibility is defined by two components: the location of opportunities within the urban area and access to those opportunities by residents (Krizek 2005). Theoretically, the location of opportunities is related to the concept of centrality as used in graph theory and network analysis (Crucitti et al. 2006; Newman 2003). In this context, centrality is a measure of how important a component of the network is relative to its neighbors and explicitly relates to the location of opportunities within a network. Access to those opportunities is determined by travel times and distances, and therefore depends both on the mode of transportation and the distance between residents and opportunities. The mode of travel and travel distance are related because higher mobility allows residents to live farther away from opportunities (Levinson and Kumar 1997; Sultana and Weber 2007). The spatial distribution of opportunities and the spatial relationship between opportunities and residents of urban areas is crucial to understanding urban structure, and hence is crucial to effectively and efficiently address planning issues in growing cities (Gifford 2005; Schrank et al. 2005).

Early attempts to put the concept of accessibility to practical use recognized these two intrinsic aspects (see Ingram 1971). Such attempts have resulted in an array of measures that quantify accessibility (Baradaran and Ramjerdi 2001; Handy and Niemeier 1997; Miller 1999). The fact that each definition emphasizes different aspects of accessibility shows that different indicators are required to fully summarize particular local characteristics of the urban infrastructure (Kwan et al. 2003). For example, using secondary literature, Baradaran and Ramjerdi (2001) identify five major theoretical approaches to quantify accessibility (travel-cost, gravity or opportunities, constraints-based, utility-based surplus, and composite approach, showing that quantified measures of accessibility are highly dependent on local conditions. But regardless of how accessibility is quantified, the objective is often to evaluate how efficient a particular aspect of the urban infrastructure is (Baradaran and Ramjerdi 2001; Handy and Niemeier 1997; Kwan and Weber 2003). At a macroscopic level, efficiency will depend on the structural characteristics of the urban system. We develop a theoretical framework largely independent of different definitions of accessibility in an attempt to understand how accessibility varies with city size, where size is measured both by population and spatial extent of the urban area.

Since accessibility is ultimately a spatial issue faced by city residents, it has been proposed that accessibility research would greatly benefit from a people-based approach, as opposed to more traditional place-based approaches (Miller 2005). While recognizing the importance of this distinction, this paper aims to understand accessibility in urban areas using a broader approach. Our analysis merges the people- and place-based approaches by considering the properties of networks that connect people to places.

This paper proposes a statistical approach inspired by recent developments of al- 
lometry and Metabolic Scaling Theory (MST) in biology (Banavar et al. 1999; Brown et al. 2004; West et al. 1997). For the purposes of assessing urban network accessibility, road networks of different sizes are studied, with an emphasis on understanding how decentralization affects transport efficiency, where efficiency is defined as minimizing transportation times and distances. This analysis shows that decentralization is an important difference between urban road networks and the biological cardiovascular networks that MST was originally developed to describe. In particular, this paper examines the relationship between macroscopic properties of urban road networks and urban area, population size and accessibility; 425 urban areas with different populations and areas are compared, rather than looking at the local structure within these cities. Based on this analysis, a theoretical framework is proposed to understand how efficiently cities of different sizes distribute traffic through road networks. By sacrificing specific details of the local movement of people within the urban areas, this approach unveils the most general set of features that determine accessibility across different cities.

\section{Cities as organisms}

The approach used in this study is based on the premise that there is some commonality in the ways that cities and organisms distribute resources through networks. In the early 1970s, Howard T. Odum (Odum 1971, 1973) proposed that flows of energy and materials in societies can be analyzed in the same way they are analyzed in organisms and ecosystems. While this approach is not completely novel (e.g. Bon 1979), the notion that societies or cities can be studied using tools from biology is increasingly relevant as ecologists study the metabolism of cities (Decker et al. 2000, 2007), the ecological footprints of cities and regions (Luck et al. 2001), and the ecological impacts of human societies (Bettencourt et al. 2007; Vitousek et al. 1986, 1997; Wackernagel et al. 2002).

Just as blood vessels of the vascular network distribute energy and materials to cells in an organism, road networks distribute energy, materials and people in an urban area. Recently, Metabolic Scaling Theory (Brown et al. 2004) has explained a long-known pattern that particular attributes of species scale non-linearly with body size (see Peters 1983, for a summary). Interestingly, similar non-linear patterns have been described for cities, and no satisfactory explanation has been advanced to date (but see Batty and Longley 1994; Makse et al. 1998). MST shows how aspects of the vascular network necessarily change systematically with the volume of the organism that is supplied by the network (Banavar et al. 1999; West et al. 1997). Further, MST shows how network properties determine many other properties of organisms, such as metabolic rate (the rate of flux of energy and materials in an organism), growth (Moses et al. 2008; West et al. 2001), and reproductive rate and lifespan (West and Brown 2005). Thus, by 
understanding properties of the distribution networks within organisms, it is possible to understand an important set of primary constraints on how organisms function (Brown and West 2000).

According to MST, energy is distributed in organisms by networks that are characterized by hierarchical branching and "space-filling," meaning that resources are delivered to cells throughout the organism. For example, in the systemic cardiovascular network, blood flows from a central pump (the heart), through the aorta to progressively lower-capacity arteries and finally to capillaries. A similar hierarchical network of veins returns blood back to the heart. Such networks allow flow of blood between a central heart and cells throughout the organism.

Urban road networks are similar to the cardiovascular network in that they form hierarchical branching structures that deliver traffic throughout the the city. Freeways connect to boulevards which connect to lower-capacity surface streets with the design goal of distributing people and cars throughout the city. In addition to these similarities, this study highlights an important difference between road networks and cardiovascular networks: road networks have no central point analogous to the heart that is the source and destination of all flow in the network. The similarities and differences between cardiovascular systems and road networks are outlined in Table 1. In addition to the geometric similarities between the two systems, there is an important conceptual similarity in function. As the main form of transportation of energy and materials in a city, road networks constrain the "metabolism" of a city, which we define as the rate at which energy, materials and people are moved through the city. Accessibility is a particularly significant measure of urban metabolism because it measures the rate at which people are transported to destinations. The framework proposed here allows researchers to focus on the rate at which cars arrive at their destinations as an index of both accessibility and urban metabolism, with important implications for the organization of urban areas.

\begin{tabular}{lll}
\hline & Cities & Organisms \\
\hline Metabolism & $\begin{array}{l}\text { Car delivery rate (to } \\
\text { destinations })\end{array}$ & Oxygen delivery rate to cells \\
System size & City area $\left(A_{\text {city }}\right)$ & Body Volume $\left(V_{\text {org }}\right)$ \\
Network size & Road surface area $\left(A_{\text {road }}\right)$ & Cardiovascular volume $\left(A_{\text {net }}\right)$ \\
Density & Population density $(\rho)$ & Cell density $(\rho)$ \\
Predicted scaling & $A_{\text {road }} \propto \rho A_{\text {city }}^{3 / 2}$ & $V_{\text {net }} \propto \rho V_{\text {org }}^{4 / 3}$ \\
\hline
\end{tabular}

Table 1: Comparison of cities and biological systems 


\section{Metabolic Scaling Theory}

The efficient delivery of energy and materials to every cell within an organism is a major biological challenge. MST posits that in order to ensure survival and reproduction through evolutionary time scales, organisms have evolved vascular networks that balance competing constraints of maximizing metabolic rate, minimizing transportation distances and times, and minimizing the length and construction of the transportation network itself. MST shows that centralized, space-filling, hierarchical resource distribution networks, such as the circulatory systems of mammals, are solutions to this optimization problem because they deliver blood from a central heart to all parts of the organism as efficiently as possible. The geometric properties of such networks generate a series of scaling patterns that have long been observed in biology (Calder 1983; West et al. 1997).

It is well known that most rates in biology scale with the size of organism (Peters 1983), meaning that they are proportional to mass raised to some exponent. Biological rates can be expressed as $r \propto M^{b}$ where $r$ is the rate of interest (e.g. metabolism, geographic distribution range, heart rate among others), $M$ is body mass, and $b$ is the scaling exponent. MST explains that $b=3 / 4$ for metabolic rate (Peters 1983) because of the scaling properties of distribution networks (West et al. 1997). MST also uses network scaling properties to explain scaling for blood circulation time, $b=1 / 4$. Further, MST shows that other biological properties such as lifespan, speciation rate and growth rates are also indirectly dependent on the rate at which networks deliver resources to cells, and explains why many attributes of organisms show $1 / 4$ power scaling with $M$. While biologists typically study how rates scale with $M$, the mathematical development of the theory focuses on why rates vary systematically with organism volume, $V_{\mathrm{org}}$. Since the density of cells in organisms does not change systematically with mass, $M$ and $V_{\text {org }}$ are linearly related. The key result of MST describes how the volume of the delivery network $\left(V_{\text {net }}\right)$ scales nonlinearly with the volume of the organism $\left(V_{\text {org }}\right)$ and linearly with the density of delivery sites $(\rho)$ within the organism.

$$
V_{\text {net }} \propto \rho V_{\text {org }}^{4 / 3}
$$

A detailed derivation of Eq. 1 based on fractal geometry is given in West et al. (1997). This result can be understood intuitively by realizing that as the volume of the organism grows, there are simultaneously more cells, and the average distance between the heart and any cell increases. The increase in distance per cell is proportional the radius of the organism, or $V_{\text {org }}^{1 / 3}$, and the number of cells is proportional to $V_{\text {org }}$; multiplying these two terms gives the $4 / 3$ exponent in Eq. 1. Biology imposes a further constraint that $V_{\text {net }}$ is a constant percentage of $V_{\text {org }}$ so that $V_{\text {net }} \propto V_{\text {org }}$. Combining this constraint with the constraint that metabolism $(B)$ is linearly related to the number 
of network delivery sites generates the relationship $B \propto V_{\text {org }}^{3 / 4}$, and the scaling of other biological rates and times follow. Thus, MST explains a number of long-standing observations in biology as resulting from the scaling properties of distribution networks. This is the conceptual framework used herein to explain the allometric relationships between urban road networks and city population and area.

\section{Using MST to characterize road networks}

Translating MST predictions to road networks is straightforward under the assumption that roads are delivering cars to and from the city center. The 4/3 exponent in Eq. 1 reflects the 3-dimensional structure of organisms. The exponent in Eq. 1 can be generalized to a system in any dimension, by $S_{\text {net }} \sim S_{\text {sys }}^{b}$ where $S_{\text {net }}$ is the size of the network and $S_{\text {sys }}$ is the size of the system in dimension $D$, and $b=(D+1) / D$. In particular, for a 2-dimensional city area:

$$
A_{\text {road }} \propto \rho A_{\text {city }}^{3 / 2} .
$$

Thus, MST predicts that the surface area of the road network (measured in lane miles) scales linearly with population density, but non-linearly with city area. Thus, as cities increase in spatial extent, they will require proportionally more lane miles per unit city area (accounting for differences in traffic velocity changes the predictions only slightly). It is possible to rearrange Eq. 2 using $\rho=N / A_{\text {city }}$ where $N$ is population size to show the expected relationship between road network area $\left(A_{\text {road }}\right)$ and city area $\left(A_{\text {city }}\right)$ using MST as a framework, so that

$$
A_{\text {road }} \propto N A_{\text {city }}^{1 / 2} .
$$

West et al. (1997) give a thorough but complicated derivation of Eq. 3 based on the geometry of fractal branching networks; this paper gives a more intuitive explanation (see also Banavar et al. 1999). To simplify the explanation used in this study, the analysis begins with circular cities and an assumption of uniform population density and constant velocity on roads (Figure 1). These assumptions are not meant to be realistic, but altering them only changes scaling constants, not the scaling exponents that are the focus of the present analysis. The assumptions are addressed further in the discussion below.

Figure $1 \mathrm{a}$ shows a cartoon of a small city with population $N$. Figure $1 \mathrm{~b}$ shows a city twice as large but with more than three times the number of people, $N$. The roads are built to follow the MST model: each individual has one lane that leads from their house to the city center, like the leaves connected to the trunk of a tree. Here, the only destination is the city center. Clearly, the length of road necessary to connect a location 

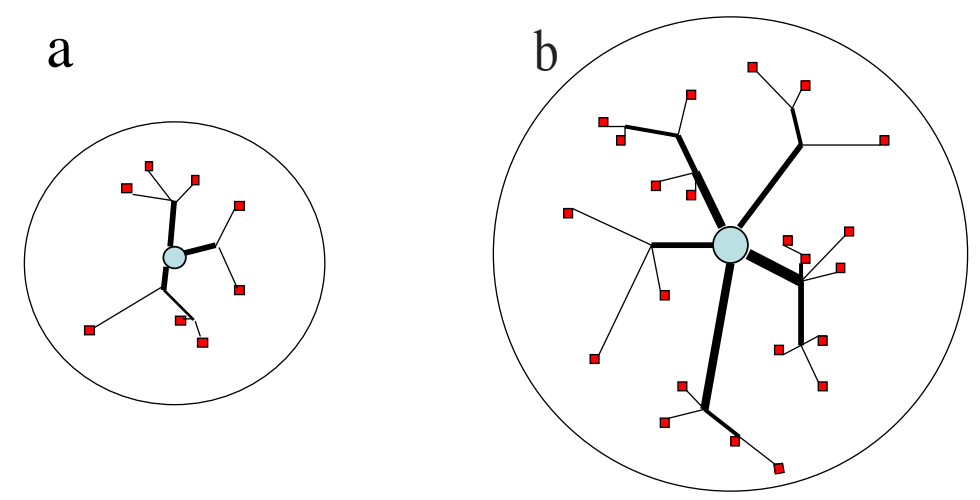

Figure 1: Model showing road network of a centralized city. Note that city (a) has half the area of city (b).

on the city perimeter to the city center is the radius of the city, $R$, where, by definition $R \propto A^{1 / 2}$. Further, the average distance from a resident to the center of the city is also proportional to $R$, regardless of the average population density of residents. Thus, in this simple centralized model, the average amount of road each person requires to reach the center of the city is proportional to $A_{\text {city }}^{1 / 2}$. At this point, another assumption of MST is invoked: the network should be capacity-preserving in order to maintain a constant flow rate through the network. In other words, to avoid congestion, a road that connects two smaller roads must have twice as many lanes. Alternatively, this can be pictured as separate roads, each one lane wide, going from the city center to each residence. Then, given $N$ residences, the total lane miles $\left(A_{\text {road }}\right)$ is proportional to $N A_{\text {city }}^{1 / 2}$ (Eq. 3). In this example the large city has both a higher population density and a larger area. The per capita lane miles are greater in the larger city only because of its larger area; per capita lane miles are unaffected by density.

With an understanding that the right-hand side of Eq. 3 simply represents the population size multiplied by the average distance to destinations, an alternative model may be considered. Figure 2 shows two cities with the same $A_{\text {city }}$ and $N$ as in Figure 1. However, instead of a fully centralized model of traffic flow, this example assumes a fully decentralized model. Destinations (circles) have been added throughout the city; for simplicity, the density of destinations has been set as equal to the population density (although the relationship between facility density and population density may not be precisely linear; see Gastner and Newman 2006b and Banerjee pers. comm.). 
This model assumes that people travel to the closest destinations, for example, the closest gas station, grocery store, bank, etc. (In Figure 1 they travel to the closest two destinations). Then the average distance $(d)$ between residents and the destinations to
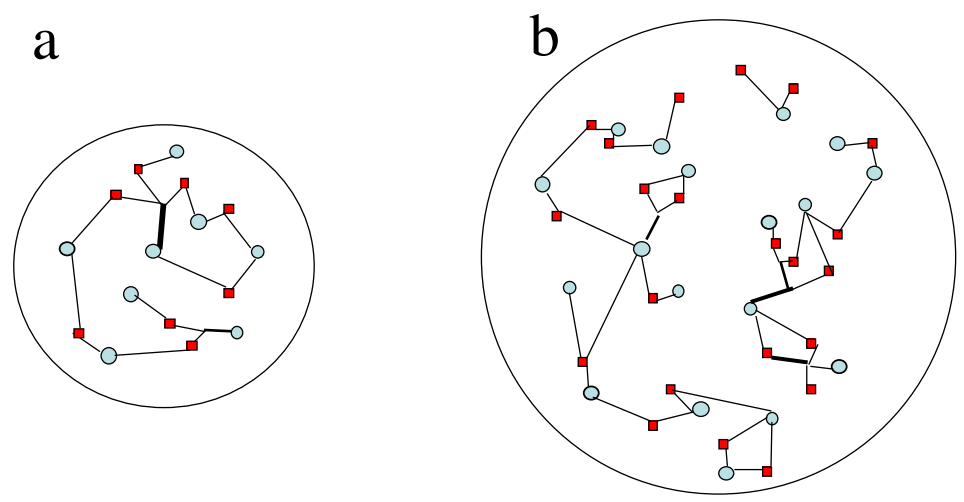

Figure 2: Model showing road network of a decentralized cities of different sizes. Note that city (a) is half the size of city (b).

which they travel is independent of $A_{\text {city }}$ but dependent on density ( $\left.\rho\right)$. In particular, from dimensional analysis, $d=\rho^{-1 / 2}$. This relationship can be seen in Figure 2. Here, $d$ is independent of $A_{\text {city }}$ but decreases as $\rho$ increases. In contrast with Figure 1, Figure 2 shows that the larger city has a lower per capita travel distance. As in Eq. 3, $A_{\text {road }}$ is proportional to $N$ times $d$, so that $A_{\text {road }} \propto N \rho^{-1 / 2}$. Since, by definition, $\rho=N / A$, this can be written as

$$
A_{\text {road }} \propto\left(N A_{\text {city }}\right)^{1 / 2}
$$

At this point, two alternative models of traffic flow are available: Equation 3 is based on MST and a completely centralized model of traffic flow; Equation 4 is based on a completely decentralized model of traffic flow. These are obviously extremes of a continuum of possible traffic scenarios, which can be used to assess where along the continuum real road networks and real traffic patterns fall.

Equations 3 and 4 are now used to test the hypothesis that urban areas and organisms exhibit similar scaling relationships. By addressing the ways in which urban road network scaling differs from scaling in biological systems, this study attempts to establish an explicit relationship between city size and accessibility. In particular, this analysis considers how road network design influences per capita travel distance, time delay, and minimization of built lane miles, as well as how variation in city spatial 
extent, population density and network geometry affect these metrics across different cities of different sizes. Variation in per capita travel distance across cities is a primary focus, as the most important component of travel cost and accessibility (Hansen 1959; Levinson and Kumar 1997). Road networks are assumed to be 2-dimensional; although some cities have population housed in tall buildings, these cases are reflected in population density. Ultimately, this work suggests how spatial extent and population density interact with aspects of urban design to alter accessibility in different cities.

\section{Data and methods}

Data for this study include lane miles and daily vehicle miles driven for 425 urban areas with population sizes ranging from $5 \times 10^{3}$ to over $3.5 \times 10^{7}$ in 2004 across the United States. Urban boundaries are circumscribed to Metropolitan and Micropolitan Statistical Areas as defined by the U.S. Office of Management and Budget for 2005 (http://www.census.gov/population/www/estimates/metroarea.html). A metro area contains a core urban area of 50,000 or more population, and a micro area contains an urban core of at least 10,000 (but less than 50,000) population. These data are collected by the Federal Highway Administration (FHWA) in its Highways Statistics Annual Reports published by the U.S. Department of Transportation (FHWA 2005). Additionally, data on commuting times for 314 urban areas were obtained from the American Community Survey available on the Census Bureau's American FactFinder site (http://www.census.gov/acs/www/). Because data are submitted by the states and may be collected with different methodologies that may introduce errors, the FHWA analyzes each state for consistency against its own past years of data and also against other state and federal data. The impact of such inconsistencies is expected to be insignificant in the present analysis, given the number of urban areas analyzed and the wide range of population sizes employed in this research. Moreover, to the authors' knowledge, this data set contains the best available data on highways, motor vehicles, and driving statistics across U.S. cities.

All variables used were $\log _{10}$-transformed and compared using Standardized Major Axis regression (SMA, also known as type II-regression or reduced major axis; see Warton et al. 2006 for further details on why SMA is the appropriate statistical tool for this type of analysis). Although Ordinary Least-Square regression is traditionally used, SMA is most appropriate when variables cannot be categorized as dependent and independent, or when both variables are suspected to include measurement errors. Harrisonburg (Virginia) was removed from the commuting time analysis due to inconsistencies with respect to overall trends shown by the remaining 313 cities.

Observed properties of urban networks are compared to centralized and decentralized predictions (Eqs. 3 and 4 ) by measuring the deviation of the slope $b$ from unity. 
In order to represent both centralized and decentralized models on the same plot, Eqs. 3 and 4 are rearranged by dividing both sides by $A_{\text {city }}^{1 / 2}$.

\section{Results}

Figure $3 \mathrm{a}$ shows the predictions for Eq. 3 versus observations, and Figure $3 \mathrm{~b}$ shows predictions from Eq. 4 versus observations. An accurate scaling prediction is indicated by a slope of 1 , which indicates agreement with the exponents in the equations. Figure
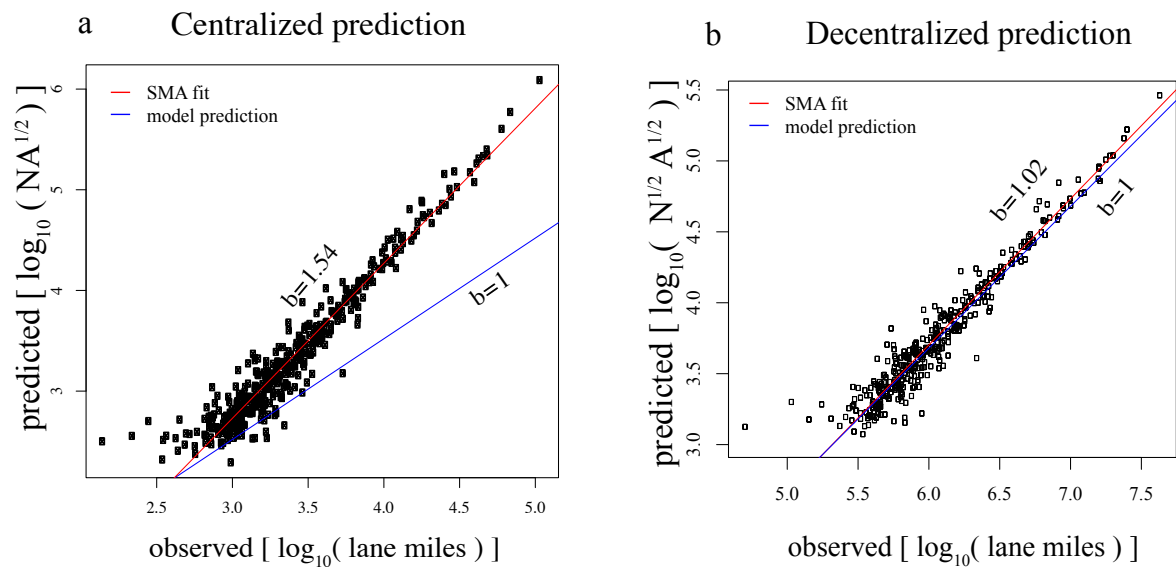

Figure 3: Double logarithmic plots of predicted scaling of network area $\left(A_{\text {road }}\right)$ as total lane miles versus predicted population $(N)$ and city area $\left(A_{\text {city }}\right)$ as the observed values based on (a) equation 3 and (b) equation 4. Observed relation based on SMA regression is shown as a red line and expected based upon our model in blue.

3a shows a slope very different from 1 , indicating that data are not consistent with the central network model. Figure $3 \mathrm{~b}$ shows an exponent indistinguishable from 1, consistent with a the decentralized model. The centralized prediction (Eq. 3) fails to capture the scaling relationship, while the decentralized prediction (Eq. 4) gives the right scaling and accounts for 93 percent of the variation in urban lane miles (Table 2).

Until this point, the analysis has focused on questions about how road networks are built. Next comes a related but different question: What distances do people actually drive as a function of city area and population density? This question can be answered using the same framework illustrated in Figures 1 and 2, modifying Eq. 3 and Eq. 4 by replacing $A_{\text {road }}$ with total miles driven, which is also reported directly by the FHWA. 
Table 2: Observed and predicted fit using Standardized Major Axis regression. Note that a slope of one is expected from our model for each situation (See Eqs. 3 and 4). $p<0.0001$ for all relations.

\begin{tabular}{lccccccc}
\hline & \multicolumn{3}{c}{$\begin{array}{c}\text { Centralized } \\
\left(\left(N A_{\text {city }}\right)^{1 / 2}\right)^{b}\end{array}$} & & \multicolumn{3}{c}{$\begin{array}{c}\text { Decentralized } \\
\left(N A_{\text {city }}^{1 / 2}\right)^{b}\end{array}$} \\
\cline { 2 - 4 } \cline { 6 - 8 } & $b$ & $95 \%(C I)$ & $R^{2}$ & & $95 \%(C I)$ & $R^{2}$ \\
Lane miles $\left(A_{\text {road }}\right)$ & 1.54 & $(1.531 .62)$ & 0.925 & & 1.02 & $(1.001 .06)$ & 0.929 \\
Miles driven $\left(A_{\text {road }}\right)$ & 1.37 & $(1.341 .40)$ & 0.961 & & 0.89 & $(0.870 .92)$ & 0.950 \\
\hline
\end{tabular}

Figure 4 shows a slightly different pattern than Figure 3. The data are consistent with neither the centralized nor decentralized model. The scaling exponent on Figure $4 \mathrm{~b}$ is 0.89 and is statistically different from the exponent of 1 in Figure $3 \mathrm{~b}$. This indicates that total miles driven scales differently than lane miles.
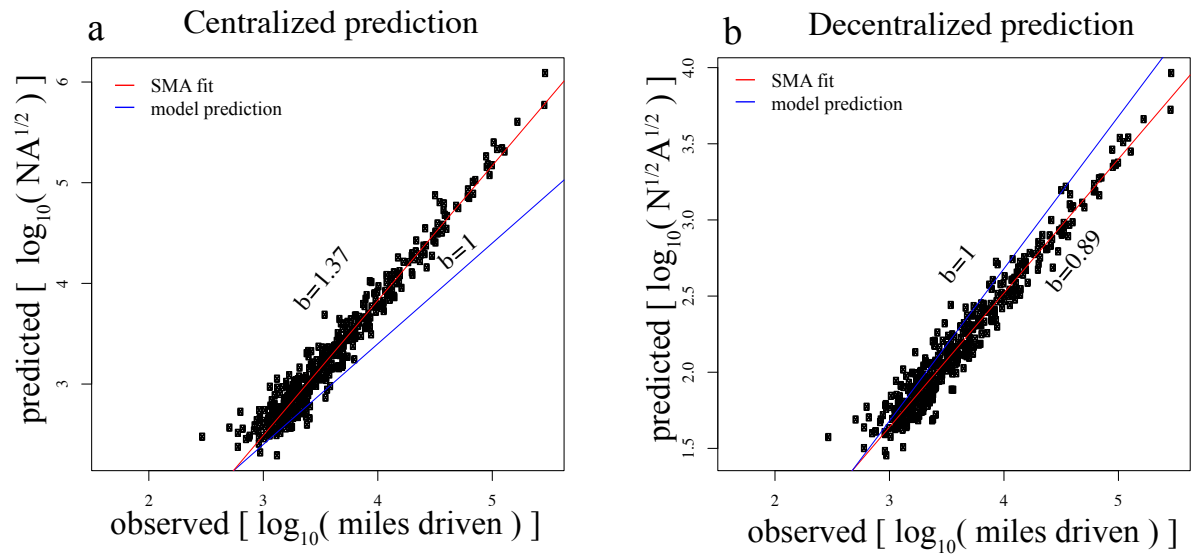

Figure 4: Double logarithmic plots of predicted scaling of network area $\left(A_{\text {road }}\right)$ as total miles driven versus predicted population $(N)$ and city area $\left(A_{\text {city }}\right)$ as the observed values based on (a) equation 3 and (b) equation 4. Observed relation based on SMA regression is shown as a red line and expected based upon our model in blue.

This is shown more clearly in Figure 6, in which the axes are rearranged in order to plot both model predictions on the same figure. Driving distances fall somewhere between the extremes of completely centralized and completely decentralized traffic flow. If travel in cities were completely centralized (as is the assumption in Eq. 3), the scaling 
exponent is predicted to be 1 ; if travel is completely decentralized, an exponent of 0.5 is predicted. The observed exponent is $0.66(95 \% C I=[0.65,0.68])$, so that miles driven reflect a network that is only partially decentralized, and as city size increases, miles driven increase faster than built lane-miles. This exponent indicates that there is not a strong signal of a centralized network; however, the significance of the SMA shows that there is some effect of city area on driving distance, so that miles driven increase faster than road capacity in larger cities. Figure $3 \mathrm{~b}$ indicates that driving distances are neither completely centralized nor completely decentralized, but somewhere in between. This suggests that some travel is to central business districts, and some is to services distributed according to population density. We hypothesized that per capita commuting traffic might be more centralized than other forms of travel. A direct test of this hypothesis would require measures of commuting distances across urban areas. Using commuting times as a proxy for commuting distance, we show that commuting is no different than travel in general shown by the nearly linear relation between commuting time and total miles driven in Figure 5 (slope $=0.98,95 \% C I=[0.95,1.001]$ ).

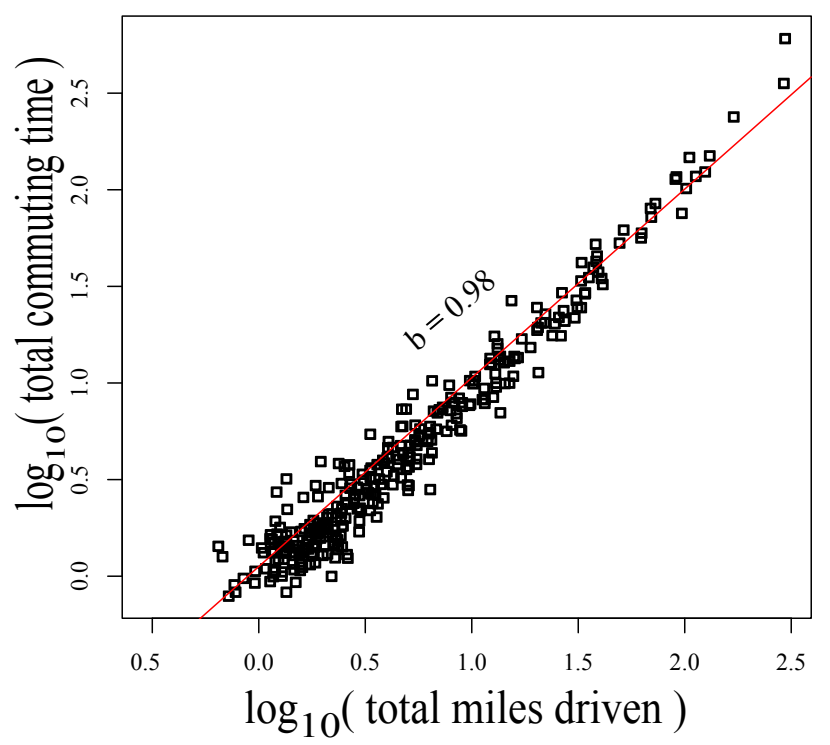

Figure 5: Double logarithmic plots of relationship between total driven miles and total commuting times in minutes. 


\section{Discussion}

This study has begun an analysis of how driving distances and road network capacities scale with city area, population size and population density. The motivation for this work grew out of long-standing empirical results and recent theoretical advances in biology that show compelling, and potentially very general, nonlinear relationships between the capacities of networks and sizes of systems in which they are embedded.

The analysis presented in this paper has shown that urban road networks are fundamentally different from vascular networks in that they are not (at least not completely) centralized. An examination of road networks across U.S. cities reveals that road networks are built as though traffic is completely decentralized - that is, as though people typically travel to their nearest store or neighbor or bank, rather than across town or to the central business district. This finding is summarized by the close linear correspondence between the decentralized prediction (Eq 4) and the data in Figure 3b, which contrasts with the poor fit of the centralized prediction (Eq 3) in Figure 3a. We found it surprising that roads are built this way.

Testing whether people actually drive the same way that roads are built (using total daily vehicle miles traveled in these same cities) revealed that that actual driving distances show a slightly different scaling than the scaling of road capacities. As shown in Figures $4 \mathrm{a}$ and $4 \mathrm{~b}$, driving patterns are not very well characterized by either model, but they are between the two extremes and somewhat closer to the decentralized prediction (see Figure 6).

This intermediate pattern may reflect a mixture of different traveling patterns. Perhaps commuting tends to be to the central business district, in which case commuting patterns would be closer to the centralized case. However, commuting times (we did not have access to commuting distances) are found to be remarkably similar to traffic in general (Figure 5). These results suggest that both commuting and recreational traffic have a mixture of both centralized and decentralized components.

\subsection{Limits of the model}

While the distribution of population density within a city is important to many aspects of urban planning (Batty and Longley 1994), the model used in this study is concerned only with average population density and is insensitive to how that density is distributed within the city. For example, if population density is a monotonically decreasing function of distance to the city center (Batty and Longley 1994), the average distance from any residence to the center remains linear with the radius of the city.

Population density serves as a surrogate for the density of destinations in this reseach. Gastner and Newman (2006a), Lammer et al. (2006) and Kühnert et al. (2006) collected data for urban areas in the U.S., and found a strong correlation between pop- 


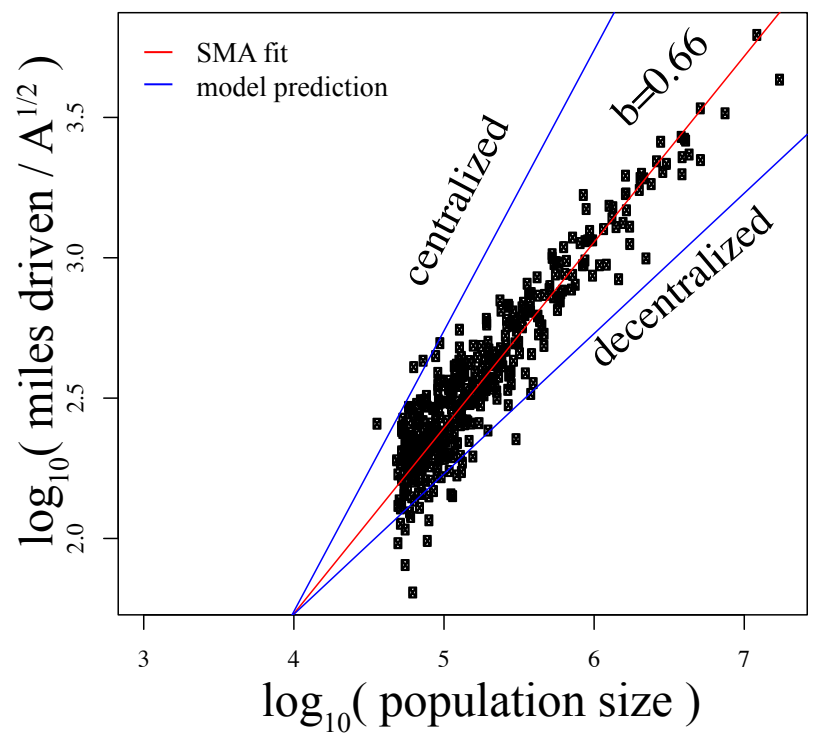

Figure 6: Road network scaling of total miles driven compared to centralized and decentralized predictions. Observed relation based on SMA regression is shown as a red line and expected based upon our model in blue.

ulation density and the density of stores and services in those cities. Interestingly, the relationship was often nonlinear, with a scaling exponent slightly less than one. Thus, approximating the density of locations with the density of residents may have introduced a slight systematic bias in the analysis. Further exploration of how the density of destinations scales with city size and population density would be of value.

Another potential source of systematic bias is the use of population size as a proxy for the number of cars on the road. The relevant question is whether there is a linear relationship between the two. To the extent that larger and more densely populated cities have more public transportation, this could introduce systematic bias. Testing a smaller subset of data (Litman 2004) in which public transportation was accounted for produced qualitatively similar results to those shown in Figures 3 and 4.

The initial assumption of constant traffic velocity is clearly violated in real cities. It matters to this model because road capacity should ideally include traffic velocity as well as lane miles. We tested whether a crude accounting for velocity would qualitatively change our results. We assumed that all highways had a velocity of 50 m.p.h. and all other roads had a velocity of 25 m.p.h.. Including this adjustment, we still found that 
road capacities closely matched the decentralized prediction (Eq. 4). Again, we think that incorporating more thorough velocity models or more detailed velocity data into this scaling framework would be worthwhile. This is a particularly interesting area of research, because velocity also slows substantially as blood flows from larger to smaller arteries within vascular networks. It would be particularly interesting to know if such slowing is a general feature of distribution networks and whether it affects the scaling of transport times.

Finally, while validation of theory is always contingent on the quality and availability of data, the generation of quantitative and specific predictions is an important tool to understand complex phenomena such as allometric scaling of urban areas. Here, this study has relied on data aggregated by the FHWA at the level of MSAs. It has been suggested that summary data such as the Highway Statistics report (FHWA 2005) might be too coarse or inherently faulty to support any conclusions because of potential heterogeneity in acquisition methodologies among states. While such inconsistencies might be of concern in some studies, we expect data inconsistencies to be negligible when comparisons are carried out across urban areas with widely different population sizes and road network areas. For example, miles driven would have to vary systematically with city size to affect our conclusions. Measurement errors would have to be large with respect to the two-orders-of-magnitude difference in population size across cities; it seems unlikely that such significant error would have gone undetected given the multiple uses and analyses carried out with this particular data set.

\subsection{Implications of the model}

This work has interesting implications for accessibility. It provides an explanation based on biological principles as to why travel distances are reduced by high population density and optimal distribution of locations (i.e. for employment, shopping, recreation, etc.). While this pattern has been studied in spatial economics (Isard 1972), it is still important to note that simple geometric constraints on transport through networks shape macroscopic patterns of cities and organisms. In larger cities, workers have access to a larger number of opportunities (Levinson and Kumar 1997), and there is increased innovative activity (Bettencourt et al. 2007), suggesting increasing returns as cities grow. However, this work shows that there are also diminishing returns since there is a larger per-capita travel cost in larger cities.

If cities were completely centralized, then large cities would have very low mobility. Even in the case of partially decentralized cities, congestion is highest among the largest U.S. cities (Schrank et al. 2005; but see Morrison and Abrahamse 1983). Congestion, in turn, has led to the emergence of polycentrism to sustain continuous growth. In fact, polycentrism may emerge from urban sprawl, as has been documented for most large urban areas (see Giuliano and Small 1991; Sultana 2000; Sultana and Weber 2007, for 
specific examples), and can be thought of as a response to increasing costs associated with the nonlinear scaling of urban network (Horner 2004).

This analysis suggests that traffic congestion is not a necessary consequence of urban sprawl. If cities are built so that travel is primarily between proximate destinations, rather than to central business districts, per capita travel is independent of city size. However, in practice there is a small but significant signal of traffic flow that depends on city size (Figure 6). This research has also uncovered a deviation between how roads are built and how people drive in different-sized cities. Built lane miles scale differently with population size than do driven miles, as indicated by the exponent of 1.0 in Figure $3 \mathrm{~b}$ and 0.89 in Figure $4 \mathrm{~b}$. This slight but statistically significant difference can mean a large disparity between built and driven miles in the largest cities, and suggests that the ratio of road capacity to travel needs is significantly lower in cities with the largest population sizes.

The scaling approach taken here necessarily makes simplifying assumptions in order to uncover basic properties of how road networks and travel distances change with city size and population density. These models, translated from other disciplines, offer valuable macroscopic perspectives on the differences between small and large cities and on how the road infrastructure and traffic might change as cities grow. Others have found that biological models offer valuable insights into how cities function (e.g. Bettencourt et al. 2007; Decker et al. 2000). The degree to which travel and transport are to centralized versus decentralized locations is an interesting difference between cities and the biological systems for which this theory was originally developed. This same distinction, travel to central versus spatially distributed destinations, is a key variable in understanding traffic in different cities. The statistics of per capita travel distances suggest that some degree of polycentrism exists in the largest cities. In biology this would be analogous to larger organisms having multiple hearts.

\section{Acknowledgements}

The authors thank Aaron Clauset, James H. Brown and Todd D. Kaplan for valuable insights and discussions that helped greatly in the development of this work. Several anonymous reviewers provided helpful comments and suggestions. MEM and HS acknowledge funding from the NIH COBRE Grant \#P20 RR018754, and MEM additionally acknowledges funding from NSF Grant \#CCF 0621900.

\section{References}

Banavar, J. R., A. Maritan, and A. Rinaldo (1999). Size and form in efficient transportation networks. Nature, 399(6732): 130-132. 
Baradaran, S. and F. Ramjerdi (2001). Performance of accessibility measures in Europe. Journal of Transportation and Statistics, 4(2/3): 31-48.

Batty, M. and P. Longley (1994). Fractal Cities. Academic Press.

Bettencourt, L. M. A., J. Lobo, D. Helbing, C. Kuhnert, and G. B. West (2007). Growth, innovation, scaling, and the pace of life in cities. Proceedings of the National Academy of Sciences, USA, 104(17): 7301-7306. doi:10.1073/pnas.0610172104.

Bon, R. (1979). Allometry in topologic structure of transportation networks. Quality and Quantity, 13(4): 307-326.

Brown, J., J. Gillooly, A. Allen, V. Savage, and G. West (2004). Toward a metabolic theory of ecology. Ecology, 85(7): 1771-1789.

Brown, J. and G. West (2000). Scaling in biology. Oxford University Press.

Calder, W. A. (1983). Ecological scaling - Mammals and birds. Annual Review of Ecology and Systematics, 14: 213-230.

Crucitti, P., V. Latora, and S. Porta (2006). Centrality measures in spatial networks of urban streets. Physical Review E, 73(3): 036125. doi:10.1103/PhysRevE.73. 036125.

Decker, E., S. Elliott, F. Smith, D. Blake, and F. Rowland (2000). Energy and material flow through the urban ecosystem. Annual Review of Energy and the Environment, 25: 685-740.

Decker, E. H., A. J. Kerkhoff, and M. E. Moses (2007). Global patterns of city size distributions and their fundamental drivers. PLoS ONE, 2(9): e934. doi:10.1371/ journal.pone.0000934.

FHWA (2005). Highway Statistics. Annual report, United States Department of Transportation, Washington, D.C. URL http://www.fhwa.dot.gov/policy/ohpi/hss/.

Gastner, M. T. and M. E. Newman (2006a). The spatial structure of networks. The European Physical Journal B - Condensed Matter, 49(2): 247-252. doi:10.1140/epjb/ e2006-00046-8.

Gastner, M. T. and M. E. J. Newman (2006b). Optimal design of spatial distribution networks. Physical Review E, 74(1): 016117. doi:10.1103/PhysRevE.74.016117.

Gifford, J. L. (2005). Access to Destinations, chapter Congestion and its Discontents, pp. $39-61$. Elsevier.

Giuliano, G. and K. Small (1991). Subcenters in the Los Angeles region. Regional Science and Urban Economics, 21(2): 163-182. doi:10.1016/0166-0462(91)90032-I.

Handy, S. and D. Niemeier (1997). Measuring accessibility: An exploration of issues and alternatives. Environment and Planning A, 29(7): 1175-1194. doi:10.1068/ a291175.

Hansen, W. G. (1959). How accessibility shapes land use. Journal of the American Institute of Planners, 25(2): 73-76.

Horner, M. W. (2004). Spatial dimensions of urban commuting: A review of major issues and their implications for future geographic research. The Professional Geogra- 
pher, 56(2): 160-173. doi:10.1111/j.0033-0124.2004.05602002.x.

Ingram, D. R. (1971). The concept of accessibility: A search for an operational form. Regional Studies, 5(2): 101-107. doi:10.1080/09595237100185131.

Isard, W. (1972). Location and space-economy. MIT Press, Cambridge, MA.

Krizek, K. J. (2005). Access to Destinations, chapter Perspectives on Accessibility and Travel, pp. $109-130$. Elsevier.

Kühnert, C., D. Helbing, and G. B. West (2006). Scaling laws in urban supply networks. Physica A: Statistical Mechanics and its Applications, 363(1): 96-103. doi: 10.1016/j.physa.2006.01.058.

Kwan, M., A. Murray, M. O'Kelly, and M. Tiefelsdorf (2003). Recent advances in accessibility research: Representation, methodology and applications. Journal of Geographical Systems, 5(1): 129-138. doi:10.1007/s101090300107.

Kwan, M. and J. Weber (2003). Individual accessibility revisited: Implications for geographical analysis in the twenty-first century. Geographical Analysis, 35(4): 341-354.

Lammer, S., B. Gehlsen, and D. Helbing (2006). Scaling laws in the spatial structure of urban road networks. Physica A: Statistical Mechanics and its Applications, 363(1): 89-95. doi:10.1016/j.physa.2006.01.051.

Levinson, D. M. and A. Kumar (1997). Density and the journey to work. Growth and Change, 28(2): 147-172. doi:10.1111/j.1468-2257.1997.tb00768.x.

Litman, T. (2004). Evaluating rail transit criticism. Victoria Transport Policy Institute, October.

Luck, M., G. Jenerette, J. Wu, and N. Grimm (2001). The urban funnel model and the spatially heterogeneous ecological footprint. Ecosystems, 4(8): 782-796. doi: 10.1007/s10021-001-0046-8.

Makse, H. A., J. S. Andrade, M. Batty, S. Havlin, and H. E. Stanley (1998). Modeling urban growth patterns with correlated percolation. Physical Review E, 58(6): 7054-7062. doi:10.1103/PhysRevE.58.7054.

Miller, H. (1999). Measuring space-time accessibility benefits within transportation networks: Basic theory and computational procedures. Geographical Analysis, 31(2): $187-212$.

Miller, H. J. (2005). Access to Destinations, chapter Place-based versus people-based accessibility, pp. 63-89. Elsevier.

Morrison, P. A. and A. F. Abrahamse (1983). Is population decentralization lengthening commuting distance? Population and Policy Review, 2: 189-206. doi: 10.1007/BF00141252.

Moses, M. E., C. Hou, W. Woodruff, W. G. B, J. C. Nekola, and J. H. Brown (2008). Revisiting a model of ontogenetic growth: Estimating model parameters from theory and data. American Naturalist, 171: 632-645. doi:10.1086/587073.

Newman, M. E. J. (2003). The structure and function of complex networks. SIAM 
Review, 45(2): 167-256. doi:10.1137/S003614450342480.

Odum, H. (1971). Environment, power, and society. Wiley-Interscience New York.

- (1973). Energy, ecology and economics. Ambio, 2(6): 220-227.

Peters, R. (1983). The Ecological Implications of Body Size. Cambridge University Press.

Schrank, D., T. Lomax, and T. T. Institute (2005). The 2005 Urban Mobility Report.

Texas Transportation Institute, Texas A \& M University.

Sultana, S. (2000). Some effects of employment centers on commuting times in the Atlanta metropolitan area, 1990. Southeastern Geographer, 41(S 225): 233.

Sultana, S. and J. Weber (2007). Journey-to-work patterns in the age of sprawl: Evidence from two midsize Southern metropolitan areas. The Professional Geographer, 59(2): 193-208. doi:10.1111/j.1467-9272.2007.00607.x.

Vitousek, P., P. Ehrlich, A. Ehrlich, and P. Matson (1986). Human appropriation of the products of photosynthesis. BioScience, 36(6): 368-373.

Vitousek, P., H. Mooney, J. Lubchenco, and J. Melillo (1997). Human domination of Earth's ecosystems. Science, 277(5325): 494. doi:10.1126/science.277.5325.494.

Wackernagel, M., N. Schulz, D. Deumling, A. Linares, M. Jenkins, V. Kapos, C. Monfreda, J. Loh, N. Myers, R. Norgaard, et al. (2002). Tracking the ecological overshoot of the human economy. Proceedings of the National Academy of Sciences, 99(14): 9266-9271. doi:10.1137/S003614450342480.

Warton, D., I. Wright, D. Falster, and M. Westoby (2006). A review of bivariate line-fitting methods for allometry. Biological Reviews, 81: 259-291. doi:10.1017/ S1464793106007007.

West, G. B. and J. H. Brown (2005). The origin of allometric scaling laws in biology from genomes to ecosystems: Towards a quantitative unifying theory of biological structure and organization. Journal Of Experimental Biology, 208(9): 1575-1592. doi:10.1242/jeb.01589.

West, G. B., J. H. Brown, and B. J. Enquist (1997). A general model for the origin of allometric scaling laws in biology. Science, 276: 122-126. doi:10.1137/ S003614450342480.

(2001). A general model for ontogenetic growth. Nature, 413(6856): 628-631. doi:10.1038/35098076. 